\title{
Pharmacokinetics of Anti-epileptic Drugs and their Clinical Significance
}

\author{
SVEIN I. JOHANNESSEN
}

The National Centre for Epilepsy, Norway

\begin{abstract}
Summary
The serum concentration achieved and maintained following the administration of a fixed drug dosage is a direct consequence of the interactions of a wide variety of interrelated processes, including drug absorption, distribution, metabolism, and excretion, and the physiological status of the patient. These interrelationships are reviewed with specific reference to the major anti-epileptic drugs, phenobarbitone, phenytoin, sodium valproate, and carbamazepine, as well as a new first-line antiepileptic, oxcarbazepine.

Both older drugs, such as phenobarbitone and phenytoin, and newer drugs, such as carbamazepine (CBZ) and sodium valproate, have been studied extensively over the past years giving valuable information for drug treatment. An important feature of oxcarbazepine (OXC), which was developed through minimal changes in the structure of $\mathrm{CBZ}$ in order to improve on the tolerability of CBZ without sacrificing efficacy, is that its metabolites do not include the 11-epoxide which has been implicated in the side-effects of CBZ. In man, OXC is metabolized to a monohydroxy derivative which has independent anti-epileptic properties. OXC seems to lack several disadavantageous pharmacokinetic properties common to other major anti-epileptic drugs. OXC does not influence its own metabolism after repeated administration, in contrast to the auto-induction displayed by CBZ. The metabolism of OXC is not influenced by antiepileptic co-medication and does not influence the kinetics of other antiepileptic drugs - or if it does, then to a lesser extent than CBZ.
\end{abstract}

\section{Introduction}

One of the objectives of pharmacokinetics is to describe the phenomena involved in drug disposition and response. Application of the basic principles in therapeutic drug monitoring has given rise to the discipline of clinical pharmacokinetics.

Several factors influence the serum levels and thus the clinical efficacy of anti-epileptic drugs. The serum concentration achieved and maintained following the administration of a fixed drug dosage is a direct consequence of 
the interactions of a wide variety of interrelated processes, including the absorption, distribution, metabolism, and excretion of the drug, and the physiological status of the patient. The study of these interrelationships forms the basis of pharmacokinetics. Important drug factors are chemical constitution, drug formulation, bioavailability, dosage schedules, timing of blood sampling, and drug interactions. Patient-specific factors include: gastrointestinal function, which can influence the absorption of a drug; serum proteins, body weight, and pregnancy, all of which affect the distribution of a drug; hepatic function, which - if compromised - alters the metabolism of a drug; and renal function, which influences the excretion of a drug. Also of great importance are genetic or constitutional factors - which may vary with age - and patient compliance.

The monitoring of serum concentrations is a crucial aid to optimum anti-epileptic drug therapy since the relationship between serum levels on the one hand and therapeutic and toxic effects on the other has been clearly established for most drugs. However, the therapeutic ranges reported for serum concentrations are only a guideline to drug therapy, and the optimum concentration for a particular patient may not necessarily lie within the ordinary therapeutic range. It is important to remember in clinical practice that it is the patient who has to be treated, not the serum level!

Generally, fluctuations in the serum level should be kept to a minimum during any given $24 \mathrm{~h}$ period, with peak and trough values within the optimum range for the patient. To achieve this, a dose frequency should be chosen based on the biopharmaceutical formulation, the bioavailability, and the half-life of the drugs, and the medication should be given at equal time intervals when possible. The balf-life varies from one compound to another and is often influenced by co-medication. In practice, however, it is an advantage if the drugs can be given in one or two daily doses, since the noon dose is frequently forgotten or avoided in school situations or at work. Three or more doses a day are very impractical and often lead to poor patient compliance. Hence, sustained-release preparations of carbamazepine and valproate have become very useful, since they give more stable serum concentrations than conventional tablets and can be given less frequently.

Whenever possible, the blood sampling time for individual patients should be standardized to ensure comparable conditions. Ideally, the samples should be taken in the morning before the first medication of the day; in out-patients, the morning dose may be postponed a couple of hours to ensure a drug-fasting state. When toxic effects of a drug are suspected during the day, it is best to take the blood sample at the time of maximum serum concentration. However, it has to be borne in mind that the therapeutic ranges are based on trough levels.

\section{Phenytoin}

\section{Absorption, distribution, metabolism, and excretion}

Phenytoin is available in various forms. In the standard tablet form for oral administration, its absorption is rather slow and varies with the chemical form and pharmaceutical formulation employed. Intramuscular injection or rectal 
application of suppositories results in such poor absorption and efficacy that they should be avoided whenever possible. To maintain the desired serum concentration by these routes, it is often necessary to use doses 2-3 times larger than those employed in oral therapy.

The intravenous route may also be used to administer loading doses of phenytoin. For infusion purposes, however, most parenteral solutions cannot be recommended, since phenytoin readily crystallizes when the alkaline concentrate is diluted. One infusion concentrate does exist, however, which can be mixed with common infusion liquids in all proportions (Phenhydan, Desitin-Werk, Hamburg).

In serum, phenytoin is mainly bound to albumin. In adults the binding rate is approximately $90 \%$ with slight interindividual differences. In premature babies and infants below the age of 3 months it is less, as it is in patients with hepatic and renal diseases.

Phenytoin in unbound form diffuses rapidly to body tissues and fluids, including cerebrospinal fluid, saliva, and tears, and is also rapidly distributed to brain tissue, which makes it useful in the treatment of status epilepticus.

The metabolism of phenytoin is slow, and the serum concentration is therefore relatively stable during long-term treatment, but between-patient variations do occur, depending mainly on genetic differences, but also on age, sex, and body weight. The dose (in $\mathrm{mg} / \mathrm{kg}$ body weight) necessary to attain certain serum concentrations is higher in subjects of lower body weight (adults versus children). It is important to bear in mind that 5-7\% of the population in Western Europe has a particularly slow oxidative metabolism of certain drugs, which may have considerable implications - also for the dosing of phenytoin.

Phenytoin is metabolized in the liver mainly by parahydroxylation. The metabolites display hardly any anticonvulsive activity. The half-life of phenytoin in serum is usually in the order of $15-20 \mathrm{~h}$, but may vary from 7-60 h. The metabolism of phenytoin, however, is limited by the fact that, in many patients, the enzyme system involved becomes saturated in the therapeutic range of concentration. The metabolic rate is thus dependent upon factors related to the dosage (i.e. concentration-dependent kinetics). The half-life, and thus the time needed to attain steady state, increases with concentration. Therefore, stepping up the dose only slightly may lead to a large increase in the serum concentration. The metabolism of phenytoin follows zero-order (Michaelis-Menten) rather than first-order kinetics, and for this reason it would seem inappropriate to estimate any particular half-life of phenytoin.

There are considerable individual differences in the phenytoin concentrations that lead to saturation of the drug-metabolizing enzyme system. Dosage adjustment of phenytoin must therefore be carried out carefully with monitoring of the serum concentration.

\section{Drug interactions}

Interactions between phenytoin and other drugs occur rather frequently. A particularly important feature of phenytoin in this respect is that it has enzymeinducing properties which can stimulate the metabolism of other drugs, 
resulting in lower serum concentrations and reduced therapeutic efficacy. The main interactions are probably those which lead to an inhibition of phenytoin metabolism. Since phenytoin follows non-linear kinetics by virtue of its saturable metabolism, even a slight inhibition of metabolism may lead to a considerable increase in the serum concentration and pharmacological effect.

\section{Phenobarbitone}

\section{Absorption, distribution, metabolism, and excretion}

Phenobarbitone may be given by oral, intravenous, and intramuscular routes. However, i.v. injection entails a risk of respiratory depression, and distribution of the drug to brain tissue is also relatively slow following i.v. administration. The rate and degree of gastrointestinal absorption following oral intake depend upon several factors, including the pharmaceutical formulation of the drug, the emptying of the stomach, and the food and drug content of the stomach. The bioavailability is almost complete $(80-90 \%)$, and peak serum concentration is usually attained $2-8 \mathrm{~h}$ after dose intake, but slower absorption has also been reported.

If more rapid absorption and effect are necessary, phenobarbitone can be given i.m. to young people and adults. The peak serum concentration is then attained within $0.5-2 \mathrm{~h}$, and the bioavailability for the two modes of administration is similar. Since the use of intramuscular injections in children is hazardous owing to possible nerve damage, this mode of administration should be used only when other routes are excluded.

Phenobarbitone is about $50 \%$ bound to serum proteins in adults, with small individual variations; in children below the age of 3 months, the protein binding rate is somewhat lower, particularly in hyperbilirubinaemia.

The metabolism of phenobarbitone is particularly slow. Variations in the steady-state concentration are therefore small within individual patients on a constant dosage. Between-patient variations are also smaller than those for phenytoin. However, monitoring of the serum concentration is necessary during long-term treatment, partly because the relationship between dose and serum concentration varies with age, sex, and interaction with other drugs. Approximately $20-40 \%$ of a given dose of phenobarbitone is excreted unchanged in the urine. The rest is slowly metabolized in the liver, none of the metabolites having any therapeutic effect. The excretion of phenobarbitone is considerably higher when the $\mathrm{pH}$ of the urine is alkaline.

The half-life of phenobarbitone in serum is $50-160 \mathrm{~h}$ in adults; in children it is shorter $(30-70 \mathrm{~h})$. The elimination follows first-order kinetics, but the possibility of saturation (zero-order) kinetics during extreme intoxication cannot be excluded.

\section{Drug interactions}

As with phenytoin, pharmacokinetic interactions with phenobarbitone are seen frequently. Phenobarbitone also has enzyme-inducing properties and may thus influence the oxidative processes for drugs in the liver or, by some 
other mechanism, alter the effect of other medication. Other drugs may also modify the effect of phenobarbitone.

\section{Carbamazepine}

\section{Absorption, distribution, metabolism, and excretion}

To date, carbamazepine is available only in oral form.

Following oral administration, the gastrointestinal absorption is slow and unpredictable both for tablets and suspension. Peak serum concentrations are usually attained $4-8 \mathrm{~h}$ after administration of conventional tablets, but have also been reported after as long as $24 \mathrm{~h}$.

Recently, several generic preparations of carbamazepine have been introduced on to the market with differing absorption characteristics, which may be of some clinical consequence. In general, a warning should be given about substituting a preparation in well controlled patients if the documentation for the generic drug is unsatisfactory.

The rate of absorption of carbamazepine is more rapid following food intake owing to a higher degree of solubility, which results in higher peak serum concentrations. The degree of absorption, however, is not influenced. On account of the special absorption characteristics displayed by carbamazepine, it takes longer to attain peak serum concentrations following administration of large doses, and the absorption is often reduced when daily doses are greater than $20-25 \mathrm{mg} / \mathrm{kg}$.

Controlled-release formulations are absorbed more slowly than conventional tablets and produce a more stable serum concentration both during the day and at night - even when given twice daily as compared with conventional tablets given three times daily.

Carbamazepine is approximately $70-80 \%$ bound to serum protein, with small interindividual differences. The binding is only influenced to a small extent by other anti-epileptic drugs. The concentration of carbamazepine in saliva, cerebrospinal fluid, and tears corresponds to the concentration of unbound drug in serum.

The metabolism of carbamazepine follows first-order kinetics and may vary considerably, which can make for a poor correlation between the dose and the serum concentration, especially in patients also receiving other anti-epileptic drugs. The half-life following administration of single doses in healthy subjects is $20-55 \mathrm{~h}$; during long-term treatment it is considerably shorter: $8-20 \mathrm{~h}$. This difference is due to auto-induction, an effect of carbamazepine which leads to increased activity of the microsomal enzymes in the liver which are responsible for its own metabolism. This may result in "time-dependent" kinetics as the elimination increases gradually during the first 3-6 weeks of treatment, necessitating a dose increase at a later stage to maintain the serum concentration. Auto-induction probably depends on the size of the dose, and on whether the patient is also being treated with other anti-epileptic drugs or with other enzyme inducers.

Carbamazepine is almost completely metabolized. Oxidation leads to formation of the main metabolite, carbamazepine-10,11-epoxide. In animal 
studies this epoxide also has an anti-epileptic effect of the same order as carbamazepine, and it is assumed also to contribute to the pharmacological effect in man.

There is a good correlation between serum concentrations of epoxide and carbamazepine in groups of patients receiving carbamazepine as the only drug. However, for practical clinical purposes, only the serum concentration of carbamazepine is measured at present, and this will continue to be the case until the importance of the epoxide has been investigated more closely.

Only about $2 \%$ of the dose is excreted as unchanged carbamazepine in urine, and the identified metabolites account for only $30 \%$, mainly as diol compounds in free or conjugated form.

\section{Drug interactions}

Like phenytoin and phenobarbitone, carbamazepine is a potent enzyme inducer and, as well as influencing the metabolism of other drugs, can also as mentioned above - stimulate its own metabolism (auto-induction). In addition, a number of drugs may change the pharmacokinetic properties of carbamazepine.

\section{Sodium valproate}

\section{Absorption, distribution, metabolism, and excretion}

Valproate may be given orally, rectally, or intravenously. Following oral administration of valproate, the degree of gastrointestinal absorption is satisfactory, whether the compound is given as a sodium salt or as an acid. The bioavailability is almost complete (96-100\%) for all formulations in common use (conventional tablets, enteric coated tablets, sustained-release tablets, capsules, and solutions). The rate of absorption, however, depends on the formulation. Usually conventional tablets and solutions give peak serum concentrations 1-2 h after administration, enteric coated tablets after 3-6 h, and sustained-release tablets after $10-12 \mathrm{~h}$. The absorption is more rapid during maintenance therapy than after single doses. It is considerably delayed if the drug is taken 2-3 $\mathrm{h}$ after a meal, which can explain why absorption tends to be considerably slower in the afternoon than in the morning.

Valproate is also rapidly absorbed following rectal application, and approximately the same serum concentrations are attained following suppositories or oral solutions used rectally as following oral medication. Rectal application, therefore, offers an alternative mode of administration when the drug cannot be given orally.

Valproate is $78-94 \%$ bound to serum proteins. The binding is concentration-dependent, and if the concentration of valproate is greater than $600 \mathrm{mmol} / \mathrm{l}$, binding may be as low as $67 \%$. The protein-bound fraction is less in patients with renal disease, chronic liver disease, and other conditions with low albumin concentration. It is doubtful whether the binding of valproate is influenced by other anti-epileptics.

Due to interindividual differences in the protein binding of valproate, 
measurement of the concentration of free drug instead of the total concentration in serum has been suggested.

The concentration in saliva correlates poorly with the serum concentrations, owing to certain physicochemical properties of the drug. In contrast to other anti-epileptics, therefore, analysis of valproate in saliva is of no practical value.

The metabolism of valproate is independent of the serum concentration and follows first-order kinetics, suggesting that there is no auto-induction or saturation of the metabolism. Due to interindividual differences in metabolism there is a poor correlation between the dose of valproate and the serum concentration, especially in patients who are also receiving other antiepileptics. The half-life of valproate in serum is $11-20 \mathrm{~h}$, and the substance is almost completely metabolized, only a small amount being found in the urine as unchanged drug.

\section{Drug interactions}

Pharmacokinetic interactions with valproate are seen frequently, and several are of major clinical significance.

While valproate inhibits the metabolism of a number of drugs, its own metabolism may also be stimulated by the enzyme-inducing activity of other anti-epileptic compounds, causing the valproate concentration to decrease. This is of considerable clinical significance, because it is often difficult to obtain satisfactory efficacy with valproate in patients also being treated with phenytoin, phenobarbitone, primidone, or carbamazepine.

These interactions with valproate make close monitoring of the serum concentrations especially important. It is noteworthy that valproate does not diminish the effect of oral contraceptives, as is the case with enzyme-inducing anti-epileptic drugs.

\section{Oxcarbazepine}

\section{Absorption, distribution, metabolism, and excretion}

Oxcarbazepine is the 10-keto derivative of carbamazepine and is rapidly and almost completely metabolized to 10,11-dihydro-10-hydroxycarbamazepine. Absorption of oxcarbazepine in humans has, therefore, been investigated on the basis of the serum concentration profile and urinary excretion patterns of this monohydroxy derivative (MHD). These investigations show that oxcarbazepine is almost completely absorbed, and that the serum concentration of MHD is comparable to that obtained when the active metabolite itself is administered. Oxcarbazepine is only available for oral use. Following a single oral dose of tablets in healthy subjects peak serum concentrations are attained within $4-6 \mathrm{~h}$. This is also the case in patients receiving long-term treatment on a twice-daily dosage schedule.

Only $38 \%$ of MHD is bound to human serum proteins compared with $67 \%$ for the parent compound. As a neutral lipophilic substance, the active metabolite of oxcarbazepine is able to diffuse rapidly through the various 
membranes and barriers of the organism. The substance also crosses the blood-brain barrier.

Although carbamazepine and oxcarbazepine are chemically similar, their biotransformation in humans differs completely. Carbamazepine is metabolized mainly by oxidative reactions, whereas oxcarbazepine undergoes metabolic reduction to the MHD, without epoxide formation.

Following a single oral dose of ${ }^{14} \mathrm{C}$-labelled oxcarbazepine in healthy subjects, $68 \%$ of total plasma radioactivity was attributable to MHD, and only $2 \%$ to unchanged oxcarbazepine. In the urine, MHD accounted for about $60 \%$ of total urinary radioactivity, about half being present as free compound and the other half as the glucuronide. Only $0.2 \%$ was present in the form of unchanged oxcarbazepine, and about $13 \%$ in the form of its direct glucuronide. About $3 \%$ was attributed to the dihydroxy metabolite. Other findings demonstrate complete excretion of hydroxy-carbamazepine and its metabolites from the human body.

Oxcarbazepine is rapidly eliminated from plasma, and its half-life is without practical relevance. The apparent terminal half-life of MHD, when administered as such, ranges from $8 \mathrm{~h}$. After the administration of oxcarbazepine, the elimination of MHD is mono-exponential, the half-life being $8-13 \mathrm{~h}$. The kinetics seem to be linear within the therapeutic dose range.

\section{Drug interactions}

Since the protein binding of MHD is only $38 \%$, there is little risk of drug-drug interactions by displacement from binding sites, as is known to be the case with highly protein-bound anti-epileptics during polytherapy.

Unlike the established anti-epileptic drugs, oxcarbazepine and its major active metabolite are cleared from the human body mainly by non-oxidative processes, including ketone reduction and O-glucuronidation. These processes do not depend on cytochrome P-450. Induction or inhibition of the cytochrome P-450 system, as is often encountered in anti-epileptic drug therapy, will have little effect on the kinetics of oxcarbazepine and its active metabolite. By the same token, there is little risk that oxcarbazepine will influence the kinetics of other drugs. Furthermore, in contrast to carbamazepine, oxcarbazepine does not influence its own metabolism after repeated administration.

\section{Concluding Remarks}

Obviously, the rational use of a drug depends on a clear understanding of that drug's pharmacokinetic properties. Knowledge of the pharmacokinetic properties of anti-epileptic drugs (see Table 1 for summary of pharmacokinetic parameters) and of factors that influence the kinetics is especially important when attempting to provide the individual epileptic patient with the most effective treatment accompanied by a minimum of side-effects. In the last decade, there have been several examples of pharmacokinetics being successfully applied to the individualization of drug therapy. This pharmacokinetic approach is particularly relevant in paediatric clinical 
TABLE 1 . Pharmacokinetic parameters of some anti-epileptic drugs.

\begin{tabular}{|c|c|c|c|c|c|c|c|}
\hline Pheny toin & $2-8$ & $0.5-0.8$ & 90 & $0.008-0.055$ & $7-60^{e}$ & $1-2$ & $40-80$ \\
\hline Phenobarbitone & $2-8$ & $0.4-1.0$ & 50 & $0.003-0.013$ & $50-160$ & 1 & $50-130$ \\
\hline Sodium valproate & $3-6^{c}$ & 0.140 .20 & $78-94^{\mathrm{d}}$ & $0.006-0.027$ & $11-20$ & $1-3^{c}$ & $300-600$ \\
\hline
\end{tabular}


pharmacology, since the pharmacokinetic characteristics of several important drugs are age-dependent.

Both older drugs, such as phenobarbitone and phenytoin, and newer drugs, such as carbamazepine and valproate, have been studied extensively over the past years giving valuable information for drug treatment. Oxcarbazepine is a new first-line anti-epileptic drug. It was developed by introducing minimal changes in the structure of carbamazepine in order to avoid the epoxide metabolite and thereby obtain a drug with similar clinical efficacy, but a better side-effect profile. In man, oxcarbazepine is metabolized to the monohydroxy derivative, which has independent anti-epileptic properties common to other major anti-epileptic drugs. The kinetics of the main metabolite appear to be linear within the therapeutic dosage range. Oxcarbazepine does not influence its own metabolism after repeated administration, in contrast to the autoinduction observed with carbamazepine. The metabolism of oxcarbazepine is not affected by anti-epileptic co-medication, and its influence - if any - on the kinetics of other anti-epileptics is less than that of carbamazepine. Furthermore, dosing recommendations to attain specific serum concentrations should be possible for oxcarbazepine therapy.

\section{Suggested Reading}

Bennett, W.M. (1988). Guide to drug dosage in renal failure. Clinical Pharmacokinetics, 15, 326-354.

Bertilsson, L., Höjer, B., Tybring, G., Osterloh, J. and Rane, A. (1980). Autoinduction of carbamazepine metabolism in children examined by a stable isotope technique. Clinical Pharmacology and Therapeutics, 27, 83-88.

Eadie, M.J. and Tyrer, J.H. (1989). "Anticonvulsant Therapy. Pharmacological Basis and Practice." Churchill Livingstone, Edinburgh.

Frey, H.H. and Janz, D. (Eds) (1985). "Antiepileptic Drugs." Springer, Berlin.

Henriksen, O. and Johannessen, S.I. (1982). Clinical and pharmacokinetic observations on sodium valproate. A five-year follow-up study of 100 children with epilepsy. Acta Neurologica Scandinavica, 65, 504-523.

Feldman, K.F., Dörhöfer, G., Faigle, J.W. and Imhof, P. (1981). Pharmacokinetics and metabolism of GP 47 779, the main human metabolite of oxcarbazepine (GP 47 680) in animals and healthy volunteers. In "Advances in Epileptology: XIIth Epilepsy International Symposium." (Eds M. Dam, L. Gram and J.K. Penry), pp. 89-96. Raven Press, New York.

Johannessen, S.I. (1981). Antiepileptic drugs: pharmacokinetic and clinical aspects. Therapeutic Drug Monitoring, 3, 17-37.

Johannessen, S.I. (1987). Monitoring drug treatment. In "Epilepsy: Progress in Treatment." (Eds M. Dam, S.I. Johannessen, B. Nilsson, and M. Sillanpää). pp. 109-123. John Wiley and Sons Ltd., Chichester.

Johannessen, S.I., Henriksen, O., Munthe-Kaas, A.W. and Salvesen, B. (1984). Serum concentration profile studies of tablets and suppositories of valproate and carbamazepine in healthy subjects and patients with epilepsy. In "Metabolism of Antiepileptic Drugs." (Eds R.H. Levy, W.H. Pitlick, M. Eichelbaum andJ.W.A. Meijer), pp. 61-71. Raven Press, New York.

Johannessen, S.I., Loyning, Y. and Munthe-Kaas, A.W. (in press). Medical treatment. General aspects. In "Epileptology-An International Perspective." (Eds M. Dam and L. Gram). Raven Press, New York. 
Knott, C. (1983). Measurement of saliva drug concentrations in the control of antiepileptic medication. In "Recent Advances in Epilepsy." (Eds T.A.Pedley and B.S. Meldrum), pp. 57-73. Churchill Livingstone, Edinburgh.

Krämer, G. and Hopf, H.C. (Eds) (1987). "Carbamazepin in der Neurologie." Thieme, Stuttgart.

Kutt, H. (1983). Effect of acute and chronic diseases on the disposition of antiepileptic drugs. In "Antiepileptic Drug Therapyin Pediatrics." (Eds P.L. Morselli, C.E. Pippenger and J.K. Penry), pp. 293-302. Raven Press, New York.

Kutt, H. (1984). Interactions between anticonvulsants and other commonly prescribed drugs. Epilepsia, 25, (Suppl. 2), S118-S131.

Kutt, H. (1985). Therapeutic monitoring of antiepileptic drugs. In "Recent Advances in Epilepsy." (Eds T.A. Pedley and B.S. Meldrum), pp. 183-205. Churchill Livingstone, Edinburgh.

Lennard, M.S., Ramsay, L.E., Silas, J.H., Tucker, G.F. and Woods, H.F. (1983). Protecting the poor metabolizer: clinical consequences of genetic polymorphism of drug oxidation. Pharmacy International, 3, 53-57.

Levy, R.H., Mattson, R.H., Meldrum, B.S., Penry, J.K. and Dreifuss, F.E. (Eds) (1989). "Antiepileptic Drugs." 3rd Edn. Raven Press, New York.

Levy, R.H., Pitlick, W.H., Eichelbaum, M. and Meijer,J.W.A. (Eds) (1984). "Metabolism of Antiepileptic Drugs." Raven Press, H. and Schmidt, D. (1985). Epilepsia, 25, 199-205.

Meijer, J.W.A. (Ed.) (1986). 1. Internationales Carbamazepin Slow-release Symposium, Zuckschwerdt, Munich.

Monaco, F., Piredda, S., Mastropaolo, C., Tondi, M. and Mutani, R. (1981). Diphenylhydantoin and primidone in tears. Epilepsia, 22, 185-188.

Morselli, P.L., Pippenger, C.E. and Penry, J.K. (Eds) (1983). “Antiepileptic Drug Therapy in Pediatrics." Raven Press, New York.

Pippenger, C.E. (1978). Pediatric clinical pharmacology of antiepileptic drugs: a special consideration. In "Antiepileptic Drugs, Quantitative Analysis and Interpretation." (Eds C.E. Pippeng: r, J.K. Penry and H. Kutt), pp. 315-319. Raven Press, New York.

Pippenger, C.E. (1989). Principles of applied clinical pharmacology: A guide to therapeutics. In "Clinical Use of Anticonvulsants in Psychiatric Disorders." (Eds R.M. Post, M.R. Trimble and C.E. Pippenger), pp. 41-70. Demos, New York.

Pippenger, C.E. (1989). Clinically significant antiepileptic drug interactions. In "Clinical Use of Anticonvulsants in Psychiatric Disorders." (Eds R.M. Post, M.R. Trimble and C.E. Pippenger), pp. 71-88. Demos, New York.

Rimmer, E.M. and Richens, A. (1988). Clinical pharmacology and medical treatment. In "A Textbook of Epilepsy." (Eds J. Laidlaw, A. Richens and J. Oxley), pp. 421-483. 3rd Edn. Churchill Livingstone, Edinburgh.

Schiwy, W. (1989). "Trileptal (Oxcarbazepine), Medical Aspects. Internal Report." CibaGeigy, Basle, Switzerland.

Shope, J.T. (1981). Medication compliance. Pediatric Clinics of North America, 28, (1), 5-22.

Shorvon, S. (1987). The treatment of epilepsy by drugs. In "Epilepsy." (Ed. A. Hopkins), pp. 229-282. Chapman and Hall, London.

Sillanpää, M. (1981). Carbamazepine. Pharmacology and clinical uses. Acta Neurologica Scandinavica, 64, (Suppl. 88). 


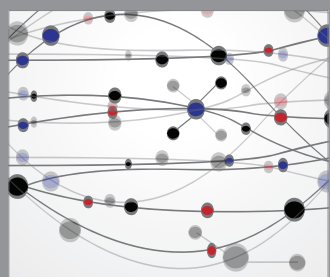

The Scientific World Journal
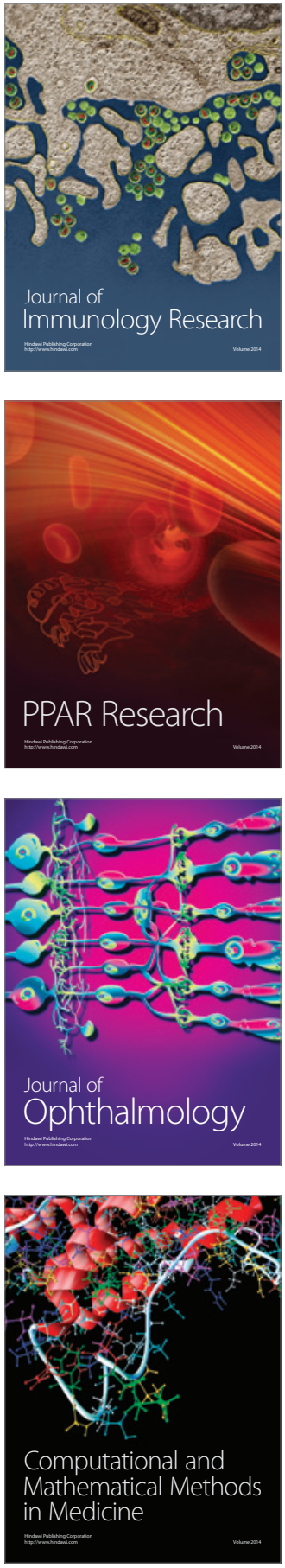

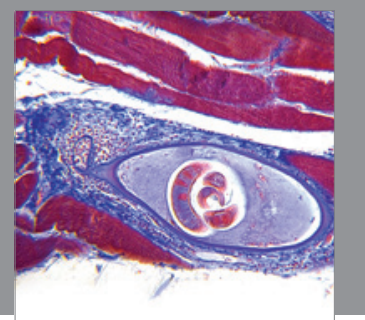

Gastroenterology

Research and Practice
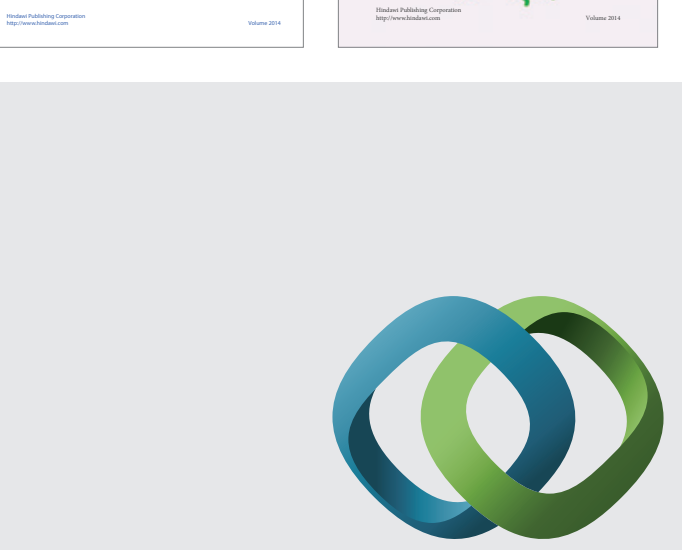

\section{Hindawi}

Submit your manuscripts at

http://www.hindawi.com
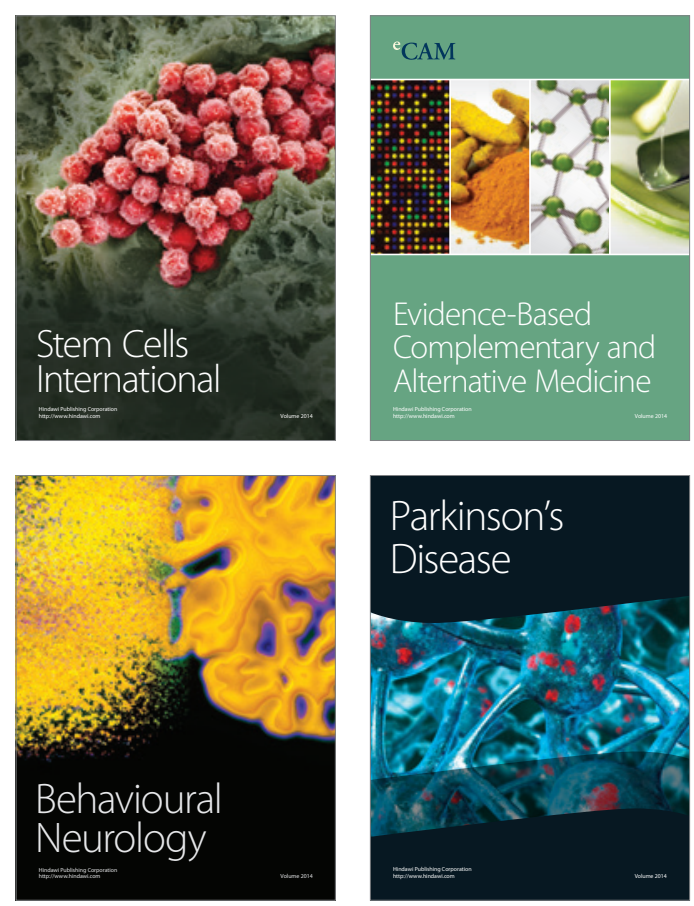

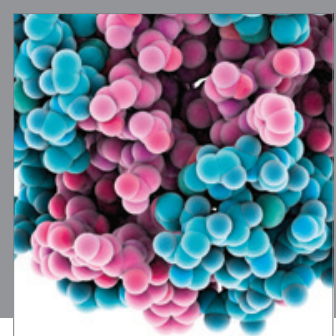

Journal of
Diabetes Research

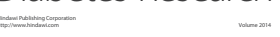

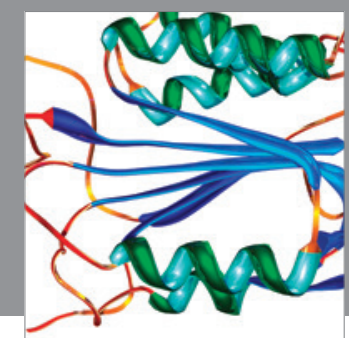

Disease Markers
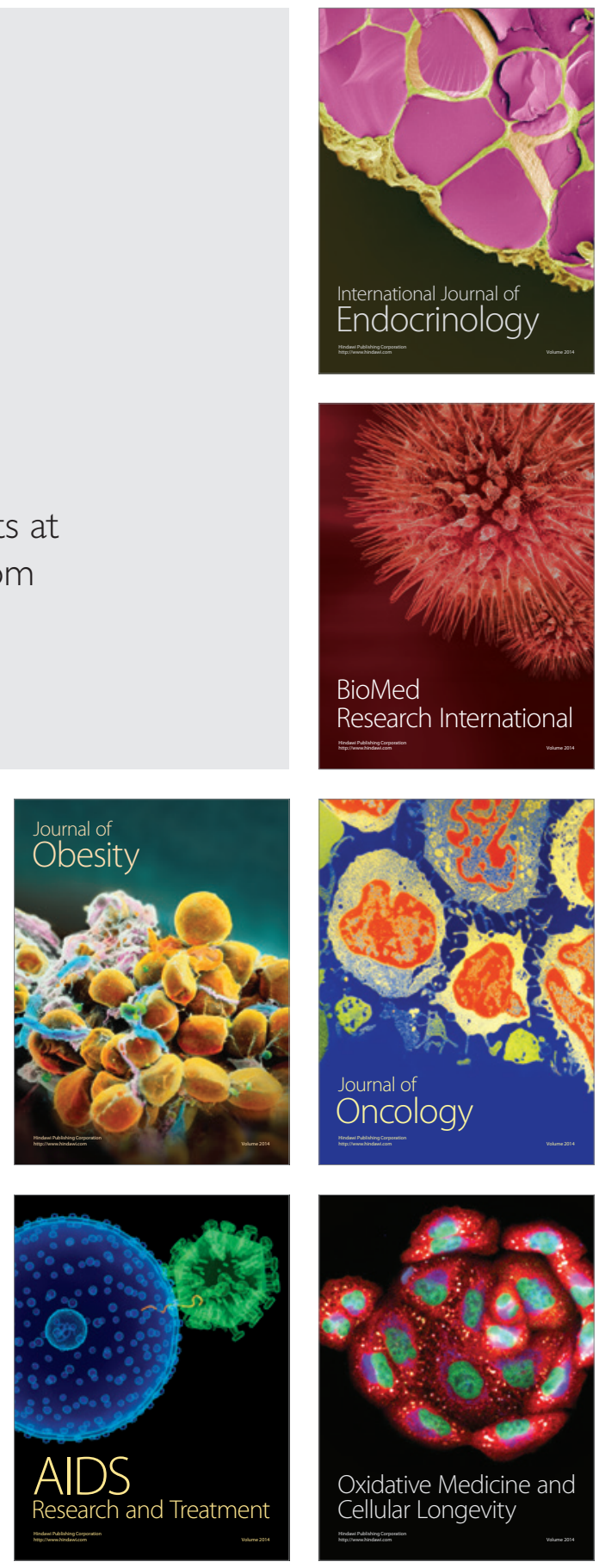